Acta Crystallographica Section E

Structure Reports

Online

ISSN 1600-5368

\section{Naphthalene-1,8-dicarboxylic anhydride: a monoclinic polymorph}

\section{Dan Zhao, ${ }^{*}$ FeiFei Li and AiYun Zhang}

Department of Physics and Chemistry, Henan Polytechnic University, Jiaozuo,

Henan 454000, People's Republic of China

Correspondence e-mail: iamzd@hpu.edu.cn

Received 6 September 2010; accepted 20 September 2010

Key indicators: single-crystal X-ray study; $T=296 \mathrm{~K}$; mean $\sigma(\mathrm{C}-\mathrm{C})=0.002 \AA$;

$R$ factor $=0.045 ; w R$ factor $=0.131 ;$ data-to-parameter ratio $=14.4$.

A new type of naphthalene-1,8-dicarboxylic anhydride, $\mathrm{C}_{12} \mathrm{H}_{6} \mathrm{O}_{3}$, was synthesized hydrothermally. Unlike the two previously reported polymorphs, which crystallize in the orthorhombic space groups $P 22_{1} 2_{1} 2_{1}$ [Shok et al. (1971). Kristallografiya, 16, 500-502; Grigor'eva \& Chetkina (1975). Kristallografiya, 20, 1289-1290] and Pbca [Shok \& Gol'der (1970). Zh. Strukt. Khim. 11, 939-940], this present structure crystallizes in the monoclinic space group $P 2_{1} / c$. In this structure, the planar [total puckering amplitude $Q=$ 0.0362 (15)] molecules lie parallel to each other along the $a$ axis.

\section{Related literature}

The previously reported polymorphs crystallize in $P 2_{1} 2_{1} 2_{1}$ (Shok et al., 1971; Grigor'eva \& Chetkina, 1975) and Pbca (Shok \& Gol'der, 1970). For puckering parameters, see: Evans \& Boeyens (1989).<smiles>O=C1OC(=O)c2cccc3cccc1c23</smiles>

\section{Experimental}

Crystal data

$\mathrm{C}_{12} \mathrm{H}_{6} \mathrm{O}_{3}$

$M_{r}=198.17$

Monoclinic, $P 2_{1} / c$

$a=3.7687$ (1) А

$b=14.5269(3) \AA$

$c=15.8083$ (3)

$\beta=94.752(2)^{\circ}$

$V=862.49(3) \AA^{3}$

$Z=4$

Mo $K \alpha$ radiation

$\mu=0.11 \mathrm{~mm}^{-1}$

$T=296 \mathrm{~K}$

$0.20 \times 0.10 \times 0.10 \mathrm{~mm}$

\section{Data collection}

Bruker APEXII CCD

diffractometer

Absorption correction: multi-scan (SADABS; Sheldrick, 1996)

$T_{\min }=0.875, T_{\max }=0.982$

Refinement

$R\left[F^{2}>2 \sigma\left(F^{2}\right)\right]=0.045$

$w R\left(F^{2}\right)=0.131$

$S=1.00$

1964 reflections

7560 measured reflections 1964 independent reflections 1201 reflections with $I>2 \sigma(I)$ $R_{\text {int }}=0.027$

Data collection: APEX2 (Bruker, 2007); cell refinement: SAINT (Bruker, 2007); data reduction: $S A I N T$; $\operatorname{program}(\mathrm{s})$ used to solve structure: SHELXS97 (Sheldrick, 2008); program(s) used to refine structure: SHELXL97 (Sheldrick, 2008); molecular graphics: SHELXTL (Sheldrick, 2008); software used to prepare material for publication: SHELXTL.

The authors acknowledge the Doctoral Foundation of Henan Polytechnic University (B2010-92, 648483) for support.

Supplementary data and figures for this paper are available from the IUCr electronic archives (Reference: HG2709).

\section{References}

Bruker (2007). APEX2 and SAINT. Bruker AXS Inc., Madison, Wisconsin, USA.

Evans, G. G. \& Boeyens, J. A. (1989). Acta Cryst. B45, 581-590.

Grigor'eva, L. P. \& Chetkina, L. A. (1975). Kristallografiya, 20, 1289-1290.

Sheldrick, G. M. (1996). SADABS. University of Göttingen, Germany.

Sheldrick, G. M. (2008). Acta Cryst. A64, 112-122.

Shok, L. N., Chetkina, L. A., Neigauz, M. G., Gol'der, G. A., Smelyanskaya, E. M. \& Fedorov, Yu. G. (1971). Kristallografiya, 16, 500-502.

Shok, L. N. \& Gol'der, G. A. (1970). Zh. Strukt. Khim. 11, 939-940. 


\section{supporting information}

Acta Cryst. (2010). E66, o2622 [doi:10.1107/S1600536810037608]

\section{Naphthalene-1,8-dicarboxylic anhydride: a monoclinic polymorph}

\section{Dan Zhao, FeiFei Li and AiYun Zhang}

\section{S1. Comment}

1,8-Naphthalenedicarboxylate (1,8-NDC), can be used as a rigid building blocks to design multiple metal-organic coordination polymers, as its multiple coordination sites, high symmetry and large conjugated structure. The singlecrystal structure of naphthalene-1,8-dicarboxylic anhydride was firstly determined by Shok and Gol'der to be a orthorhombic space group Pbca (Shok, et al., 1970). Later a $\beta$-phase was discovered with the space group $P 2{ }_{1} 2_{1} 2_{1}$ (Shok et al., 1971; Grigor'eva \& Chetkina, 1975). In this paper, a new type of naphthalene-1,8-dicarboxylic acid anhydride was hydrothermally synthesized and characterized by single-crystal X-ray diffraction with the monoclinic space group $P 2_{1} / c$.

The asymmetric unit contains only one independent molecule with the planar [total puckering amplitude $\mathrm{Q}=$ 0.0362 (15) (Evans \& Boeyens, 1989)] molecules parallel to each other along the $a$-axis (Fig. 2).

\section{S2. Experimental}

Yellow prism-shaped single crystals of Naphthalene-1,8-dicarboxylic acid anhydride were initially obtained in our attempt to prepare metal-organic coordination polymers of 1,8-NDC associated with molybdate. A mixture of 3 mmol of $\mathrm{MoO}_{3}, 2 \mathrm{mmol}$ of $\mathrm{Mn}(\mathrm{Ac})_{2}, 2.0 \mathrm{mmol} \mathrm{KOH}$ and $1.5 \mathrm{mmol}$ of Naphthalene-1,8-dicarboxylic anhydride, was sealed in a 25 $\mathrm{ml}$ Teflonlined bomb at $160^{\circ} \mathrm{C}$ for 5 days and then cooled to room temperature. A few single crystals suitable for X-ray diffraction analysis were obtained.

\section{S3. Refinement}

All of the $\mathrm{H}$ atoms were treated as riding atoms with distances $\mathrm{C}-\mathrm{H}=0.93 \AA(\mathrm{CH})$, and $U_{\text {iso }}(\mathrm{H})=1.2 U_{\text {eq }}(\mathrm{C})$. The final refinement show that the highest peak in the difference electron density map equals to $0.18 \mathrm{e} / \AA^{3}$ at the distance of $0.65 \AA$ from C5 while the deepest hole equals to $-0.17 \mathrm{e} / \AA^{3}$ at the distance of $0.61 \AA$ from $\mathrm{C} 1$. 


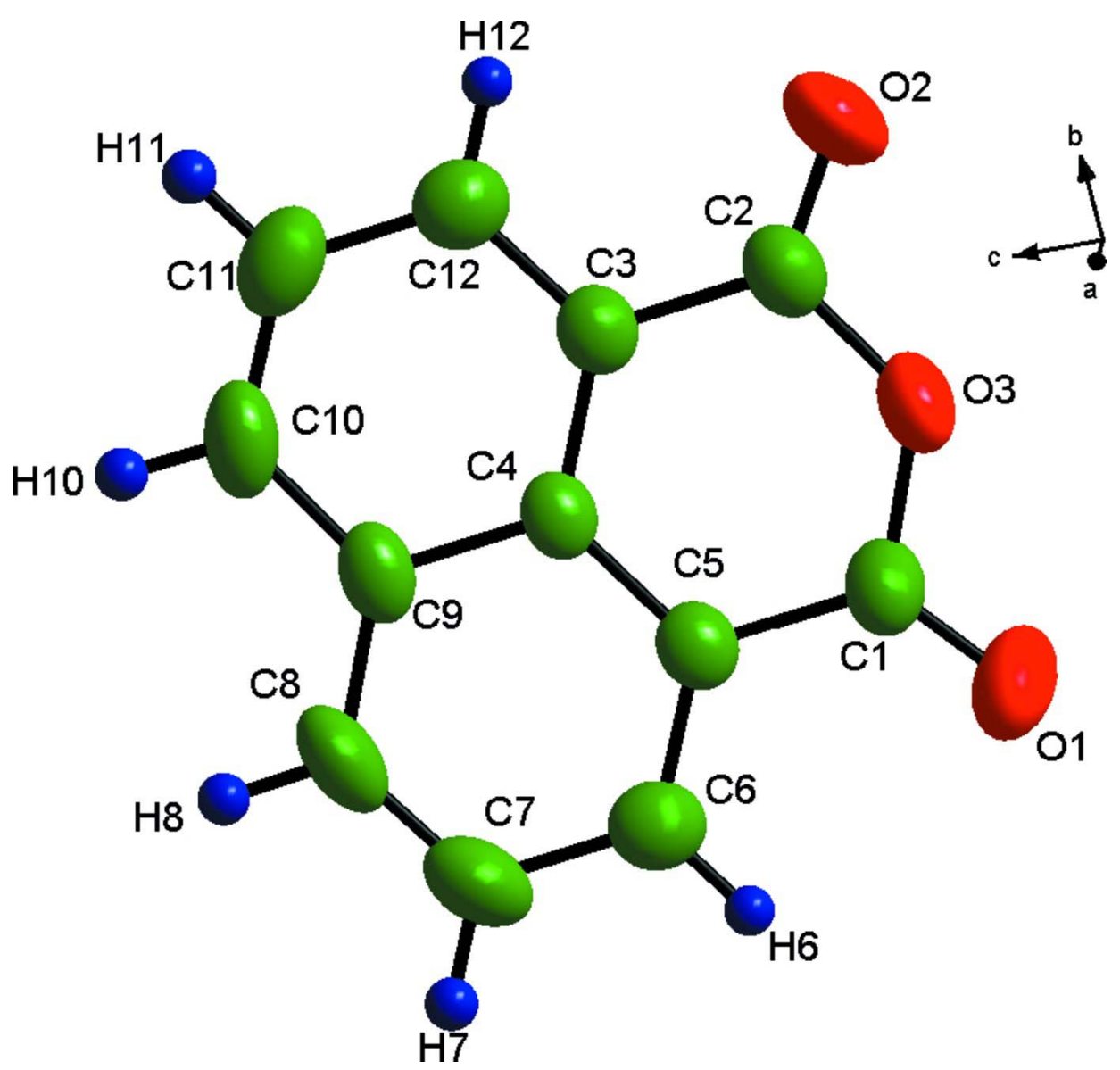

\section{Figure 1}

The molecular structure of the title compound. Displacement ellipsoids are drawn at the $50 \%$ probability level. $\mathrm{H}$ atoms are omitted for clarity. [Symmetry code: $x, y, z]$ 


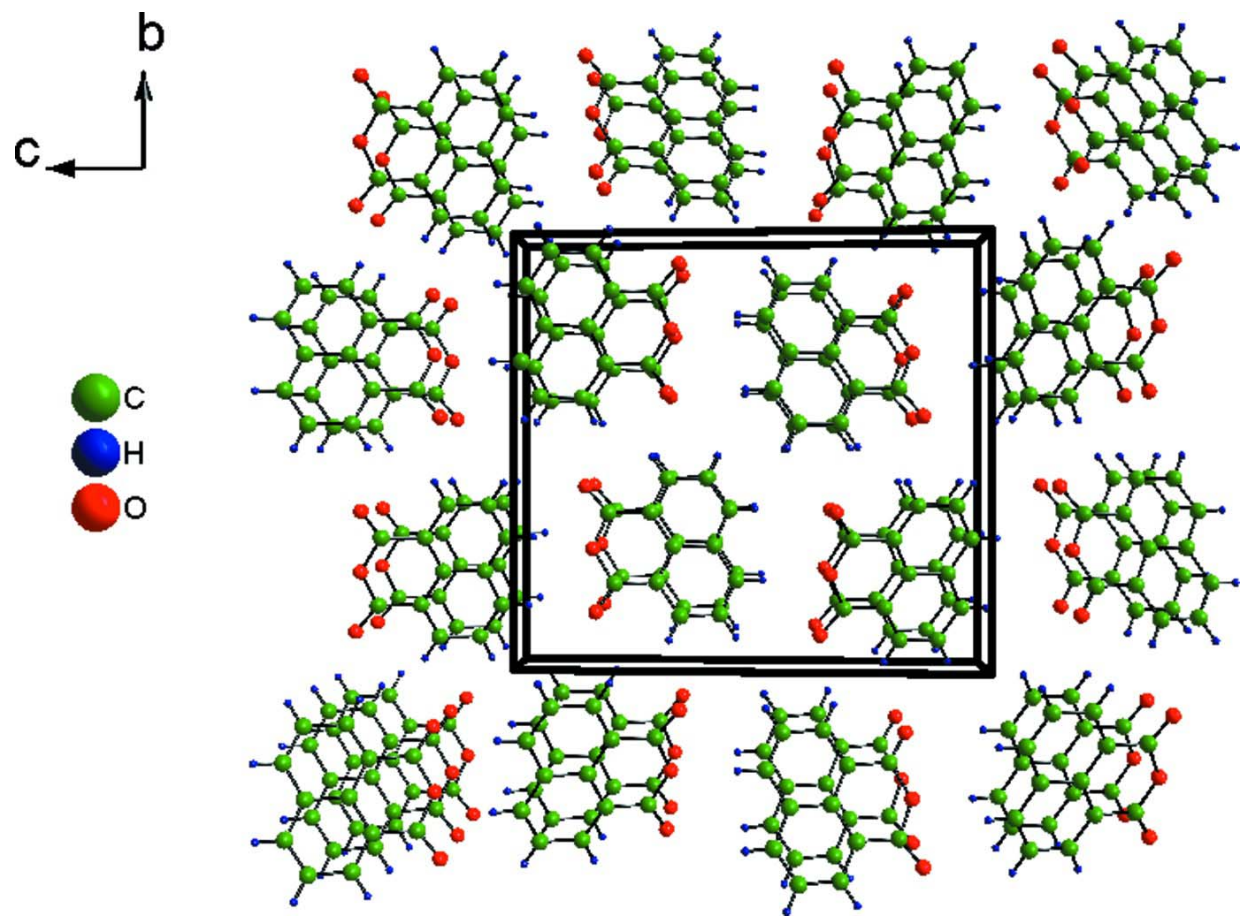

Figure 2

A packing diagram of the title compound viewed down the $a$-axis.

\section{Naphthalene-1,8-dicarboxylic anhydride}

Crystal data

$\mathrm{C}_{12} \mathrm{H}_{6} \mathrm{O}_{3}$

$M_{r}=198.17$

Monoclinic, $P 2_{1} / c$

Hall symbol: -P $2 \mathrm{ybc}$

$a=3.7687(1) \AA$

$b=14.5269(3) \AA$

$c=15.8083(3) \AA$

$\beta=94.752(2)^{\circ}$

$V=862.49(3) \AA^{3}$

$Z=4$

Data collection

Bruker APEXII CCD

diffractometer

Radiation source: fine-focus sealed tube

Graphite monochromator

$\omega$ scans

Absorption correction: multi-scan

(SADABS; Sheldrick, 1996)

$T_{\min }=0.875, T_{\max }=0.982$
$F(000)=408$

$D_{\mathrm{x}}=1.526 \mathrm{Mg} \mathrm{m}^{-3}$

Mo $K \alpha$ radiation, $\lambda=0.71073 \AA$

Cell parameters from 1938 reflections

$\theta=2.6-27.9^{\circ}$

$\mu=0.11 \mathrm{~mm}^{-1}$

$T=296 \mathrm{~K}$

Prism, yellow

$0.20 \times 0.10 \times 0.10 \mathrm{~mm}$

7560 measured reflections

1964 independent reflections

1201 reflections with $I>2 \sigma(I)$

$R_{\text {int }}=0.027$

$\theta_{\text {max }}=27.5^{\circ}, \theta_{\min }=1.9^{\circ}$

$h=-4 \rightarrow 4$

$k=-17 \rightarrow 18$

$l=-20 \rightarrow 20$ 


\section{Refinement}

Refinement on $F^{2}$

Least-squares matrix: full

$R\left[F^{2}>2 \sigma\left(F^{2}\right)\right]=0.045$

$w R\left(F^{2}\right)=0.131$

$S=1.00$

1964 reflections

136 parameters

0 restraints

Primary atom site location: structure-invariant direct methods
Secondary atom site location: difference Fourier map

Hydrogen site location: inferred from neighbouring sites

$\mathrm{H}$-atom parameters constrained

$w=1 /\left[\sigma^{2}\left(F_{\mathrm{o}}^{2}\right)+(0.0732 P)^{2}\right]$

where $P=\left(F_{\mathrm{o}}^{2}+2 F_{\mathrm{c}}^{2}\right) / 3$

$(\Delta / \sigma)_{\max }<0.001$

$\Delta \rho_{\max }=0.18 \mathrm{e} \AA^{-3}$

$\Delta \rho_{\min }=-0.17$ e $\AA^{-3}$

Special details

Geometry. All e.s.d.'s (except the e.s.d. in the dihedral angle between two l.s. planes) are estimated using the full covariance matrix. The cell e.s.d.'s are taken into account individually in the estimation of e.s.d.'s in distances, angles and torsion angles; correlations between e.s.d.'s in cell parameters are only used when they are defined by crystal symmetry. An approximate (isotropic) treatment of cell e.s.d.'s is used for estimating e.s.d.'s involving 1.s. planes.

Refinement. Refinement of $F^{2}$ against ALL reflections. The weighted $R$-factor $w R$ and goodness of fit $S$ are based on $F^{2}$, conventional $R$-factors $R$ are based on $F$, with $F$ set to zero for negative $F^{2}$. The threshold expression of $F^{2}>\sigma\left(F^{2}\right)$ is used only for calculating $R$-factors (gt) etc. and is not relevant to the choice of reflections for refinement. $R$-factors based on $F^{2}$ are statistically about twice as large as those based on $F$, and $R$ - factors based on ALL data will be even larger.

Fractional atomic coordinates and isotropic or equivalent isotropic displacement parameters $\left(\AA^{2}\right)$

\begin{tabular}{lllll}
\hline & $x$ & $y$ & $z$ & $U_{\text {iso }} * / U_{\text {eq }}$ \\
\hline C5 & $0.3898(4)$ & $0.64036(9)$ & $0.28567(8)$ & $0.0399(4)$ \\
C1 & $0.2469(4)$ & $0.64100(10)$ & $0.19687(9)$ & $0.0486(4)$ \\
C6 & $0.5444(4)$ & $0.56205(10)$ & $0.32018(9)$ & $0.0503(4)$ \\
H6 & 0.5618 & 0.5098 & 0.2868 & $0.060^{*}$ \\
C11 & $0.3060(5)$ & $0.87580(12)$ & $0.43612(10)$ & $0.0588(5)$ \\
H11 & 0.2878 & 0.9279 & 0.4696 & $0.071^{*}$ \\
C4 & $0.3603(3)$ & $0.72008(9)$ & $0.33538(8)$ & $0.0356(4)$ \\
C3 & $0.1982(4)$ & $0.80075(10)$ & $0.30145(8)$ & $0.0397(4)$ \\
C2 & $0.0514(4)$ & $0.80154(11)$ & $0.21286(9)$ & $0.0478(4)$ \\
C8 & $0.6540(4)$ & $0.63636(11)$ & $0.45452(10)$ & $0.0544(5)$ \\
H8 & 0.7441 & 0.6341 & 0.5111 & $0.065^{*}$ \\
C7 & $0.6755(4)$ & $0.56069(12)$ & $0.40523(10)$ & $0.0571(5)$ \\
H7 & 0.7788 & 0.5072 & 0.4284 & $0.068^{*}$ \\
C9 & $0.4972(4)$ & $0.71882(10)$ & $0.42179(8)$ & $0.0429(4)$ \\
O3 & $0.0835(3)$ & $0.72159(7)$ & $0.16646(6)$ & $0.0557(3)$ \\
C12 & $0.1693(4)$ & $0.87738(11)$ & $0.35104(9)$ & $0.0504(4)$ \\
H12 & 0.0594 & 0.9302 & 0.3283 & $0.061^{*}$ \\
C10 & $0.4642(4)$ & $0.79925(12)$ & $0.47005(9)$ & $0.0555(4)$ \\
H10 & 0.5534 & 0.7998 & 0.5267 & $0.067^{*}$ \\
O2 & $-0.1003(4)$ & $0.86395(8)$ & $0.17671(7)$ & $0.0747(4)$ \\
O1 & $0.2558(4)$ & $0.57887(8)$ & $0.14772(7)$ & $0.0766(4)$ \\
& & & &
\end{tabular}


Atomic displacement parameters $\left(\AA^{2}\right)$

\begin{tabular}{lllllll}
\hline & $U^{11}$ & $U^{22}$ & $U^{33}$ & $U^{12}$ & $U^{13}$ & $U^{23}$ \\
\hline C5 & $0.0368(8)$ & $0.0459(9)$ & $0.0377(8)$ & $-0.0059(7)$ & $0.0073(6)$ & $0.0044(6)$ \\
C1 & $0.0597(10)$ & $0.0486(9)$ & $0.0376(8)$ & $-0.0111(8)$ & $0.0043(7)$ & $0.0002(7)$ \\
C6 & $0.0511(10)$ & $0.0467(9)$ & $0.0544(10)$ & $-0.0002(7)$ & $0.0116(8)$ & $0.0037(7)$ \\
C11 & $0.0618(11)$ & $0.0624(11)$ & $0.0531(10)$ & $-0.0070(9)$ & $0.0104(8)$ & $-0.0178(9)$ \\
C4 & $0.0306(7)$ & $0.0447(8)$ & $0.0321(7)$ & $-0.0061(6)$ & $0.0063(6)$ & $0.0035(6)$ \\
C3 & $0.0357(8)$ & $0.0463(8)$ & $0.0376(8)$ & $-0.0043(7)$ & $0.0053(6)$ & $0.0023(7)$ \\
C2 & $0.0499(9)$ & $0.0539(9)$ & $0.0391(8)$ & $0.0002(8)$ & $0.0008(7)$ & $0.0061(7)$ \\
C8 & $0.0447(10)$ & $0.0789(12)$ & $0.0387(8)$ & $-0.0011(8)$ & $-0.0020(7)$ & $0.0195(9)$ \\
C7 & $0.0506(11)$ & $0.0605(11)$ & $0.0600(10)$ & $0.0071(8)$ & $0.0043(8)$ & $0.0210(9)$ \\
C9 & $0.0345(8)$ & $0.0618(10)$ & $0.0325(7)$ & $-0.0063(7)$ & $0.0033(6)$ & $0.0027(7)$ \\
O3 & $0.0705(8)$ & $0.0593(7)$ & $0.0354(6)$ & $-0.0055(6)$ & $-0.0075(5)$ & $0.0030(5)$ \\
C12 & $0.0495(10)$ & $0.0483(9)$ & $0.0545(10)$ & $0.0006(7)$ & $0.0103(8)$ & $0.0005(7)$ \\
C10 & $0.0524(10)$ & $0.0797(12)$ & $0.0339(8)$ & $-0.0086(9)$ & $0.0014(7)$ & $-0.0075(8)$ \\
O2 & $0.0918(10)$ & $0.0725(8)$ & $0.0572(7)$ & $0.0227(7)$ & $-0.0100(7)$ & $0.0185(6)$ \\
O1 & $0.1232(12)$ & $0.0586(8)$ & $0.0480(7)$ & $-0.0138(7)$ & $0.0075(7)$ & $-0.0131(6)$ \\
& & & & & & \\
\hline
\end{tabular}

Geometric parameters $\left(\AA,{ }^{o}\right)$

\begin{tabular}{|c|c|c|c|}
\hline $\mathrm{C} 5-\mathrm{C} 6$ & $1.3706(19)$ & $\mathrm{C} 3-\mathrm{C} 12$ & $1.3710(19)$ \\
\hline $\mathrm{C} 5-\mathrm{C} 4$ & $1.4090(18)$ & $\mathrm{C} 3-\mathrm{C} 2$ & $1.463(2)$ \\
\hline $\mathrm{C} 5-\mathrm{C} 1$ & $1.4613(19)$ & $\mathrm{C} 2-\mathrm{O} 2$ & $1.1922(17)$ \\
\hline $\mathrm{C} 1-\mathrm{O} 1$ & $1.1931(16)$ & $\mathrm{C} 2-\mathrm{O} 3$ & $1.3844(17)$ \\
\hline $\mathrm{C} 1-\mathrm{O} 3$ & $1.3900(17)$ & $\mathrm{C} 8-\mathrm{C} 7$ & $1.354(2)$ \\
\hline $\mathrm{C} 6-\mathrm{C} 7$ & $1.394(2)$ & $\mathrm{C} 8-\mathrm{C} 9$ & $1.4146(19)$ \\
\hline $\mathrm{C} 6-\mathrm{H} 6$ & 0.9300 & $\mathrm{C} 8-\mathrm{H} 8$ & 0.9300 \\
\hline $\mathrm{C} 11-\mathrm{C} 10$ & $1.351(2)$ & $\mathrm{C} 7-\mathrm{H} 7$ & 0.9300 \\
\hline $\mathrm{C} 11-\mathrm{C} 12$ & $1.400(2)$ & $\mathrm{C} 9-\mathrm{C} 10$ & $1.407(2)$ \\
\hline C11-H11 & 0.9300 & $\mathrm{C} 12-\mathrm{H} 12$ & 0.9300 \\
\hline $\mathrm{C} 4-\mathrm{C} 3$ & $1.4072(18)$ & $\mathrm{C} 10-\mathrm{H} 10$ & 0.9300 \\
\hline $\mathrm{C} 4-\mathrm{C} 9$ & $1.4197(19)$ & & \\
\hline $\mathrm{C} 6-\mathrm{C} 5-\mathrm{C} 4$ & $120.72(13)$ & $\mathrm{O} 2-\mathrm{C} 2-\mathrm{C} 3$ & $126.39(15)$ \\
\hline $\mathrm{C} 6-\mathrm{C} 5-\mathrm{C} 1$ & $119.88(13)$ & $\mathrm{O} 3-\mathrm{C} 2-\mathrm{C} 3$ & $117.26(13)$ \\
\hline $\mathrm{C} 4-\mathrm{C} 5-\mathrm{C} 1$ & $119.39(12)$ & $\mathrm{C} 7-\mathrm{C} 8-\mathrm{C} 9$ & $121.35(14)$ \\
\hline $\mathrm{O} 1-\mathrm{C} 1-\mathrm{O} 3$ & $116.57(13)$ & $\mathrm{C} 7-\mathrm{C} 8-\mathrm{H} 8$ & 119.3 \\
\hline $\mathrm{O} 1-\mathrm{C} 1-\mathrm{C} 5$ & $126.36(15)$ & $\mathrm{C} 9-\mathrm{C} 8-\mathrm{H} 8$ & 119.3 \\
\hline $\mathrm{O} 3-\mathrm{C} 1-\mathrm{C} 5$ & $117.07(12)$ & $\mathrm{C} 8-\mathrm{C} 7-\mathrm{C} 6$ & $120.70(14)$ \\
\hline $\mathrm{C} 5-\mathrm{C} 6-\mathrm{C} 7$ & $120.05(14)$ & $\mathrm{C} 8-\mathrm{C} 7-\mathrm{H} 7$ & 119.7 \\
\hline $\mathrm{C} 5-\mathrm{C} 6-\mathrm{H} 6$ & 120.0 & $\mathrm{C} 6-\mathrm{C} 7-\mathrm{H} 7$ & 119.7 \\
\hline $\mathrm{C} 7-\mathrm{C} 6-\mathrm{H} 6$ & 120.0 & $\mathrm{C} 10-\mathrm{C} 9-\mathrm{C} 8$ & $123.93(13)$ \\
\hline $\mathrm{C} 10-\mathrm{C} 11-\mathrm{C} 12$ & $120.65(14)$ & $\mathrm{C} 10-\mathrm{C} 9-\mathrm{C} 4$ & $118.02(13)$ \\
\hline $\mathrm{C} 10-\mathrm{C} 11-\mathrm{H} 11$ & 119.7 & $\mathrm{C} 8-\mathrm{C} 9-\mathrm{C} 4$ & 118.05 (13) \\
\hline $\mathrm{C} 12-\mathrm{C} 11-\mathrm{H} 11$ & 119.7 & $\mathrm{C} 2-\mathrm{O} 3-\mathrm{C} 1$ & $125.38(11)$ \\
\hline $\mathrm{C} 3-\mathrm{C} 4-\mathrm{C} 5$ & $121.58(12)$ & $\mathrm{C} 3-\mathrm{C} 12-\mathrm{C} 11$ & $119.71(14)$ \\
\hline $\mathrm{C} 3-\mathrm{C} 4-\mathrm{C} 9$ & $119.28(12)$ & $\mathrm{C} 3-\mathrm{C} 12-\mathrm{H} 12$ & 120.1 \\
\hline
\end{tabular}


supporting information

$\begin{array}{llll}\mathrm{C} 5-\mathrm{C} 4-\mathrm{C} 9 & 119.13(12) & \mathrm{C} 11-\mathrm{C} 12-\mathrm{H} 12 & 120.1 \\ \mathrm{C} 12-\mathrm{C} 3-\mathrm{C} 4 & 120.72(12) & \mathrm{C} 11-\mathrm{C} 10-\mathrm{C} 9 & 121.62(14) \\ \mathrm{C} 12-\mathrm{C} 3-\mathrm{C} 2 & 119.96(13) & \mathrm{C} 11-\mathrm{C} 10-\mathrm{H} 10 & 119.2 \\ \mathrm{C} 4-\mathrm{C} 3-\mathrm{C} 2 & 119.30(13) & \mathrm{C} 9-\mathrm{C} 10-\mathrm{H} 10 & 119.2 \\ \mathrm{O} 2-\mathrm{C} 2-\mathrm{O} 3 & 116.33(13) & & \end{array}$

